Discrete Comput Geom 31:421-433 (2004)

DOI: $10.1007 / \mathrm{s} 00454-003-2907-8$

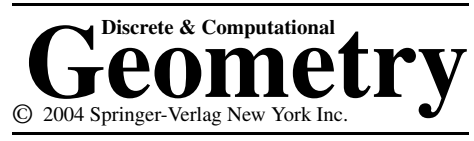

\title{
Crumpled Cube and Solid Horned Sphere Space Fillers
}

\author{
Tai-Man Tang \\ Department of Mathematics, The Chinese University of Hong Kong, \\ Shatin, New Territories, Hong Kong \\ tmtang@math.cuhk.edu.hk
}

\begin{abstract}
We construct two classes of wildly embedded space fillers of $\mathbb{R}^{3}$. First, every crumpled cube is shown to have an embedding in $\mathbb{R}^{3}$ that admits a monohedral tiling of $\mathbb{R}^{3}$. Second, a solid Alexander horned sphere with a topologically trivial interior is shown to admit a monohedral tiling of a cube and hence $\mathbb{R}^{3}$. By joining a solid horned sphere with compact polyhedral 3-submanifolds of $\mathbb{R}^{3}$ with one boundary component, we construct space fillers homeomorphic to the polyhedral submanifolds but of different embedding types. Using the suitably embedded crumpled cubes instead of a solid horned sphere, space fillers of even more different topological types can be produced.
\end{abstract}

\section{Introduction}

A tiling $\mathcal{T}$ of $\mathbb{R}^{3}$ is a countable family of closed subsets of $\mathbb{R}^{3}$, the tiles, which cover $\mathbb{R}^{3}$ without gaps or overlaps [GS]. That is, if the closed sets $T_{i}$ are the tiles, $i \in \mathbb{N}$, then $T_{i}^{\circ} \cap T_{j}^{\circ}=\emptyset$ for $i \neq j$ and $\bigcup T_{i}=\mathbb{R}^{3}$. As usual, we restrict ourselves to compact connected $T_{i}$ with $T_{i}=\overline{T_{i}^{\circ}}$. $\mathcal{T}$ is said to be monohedral if each $T_{i}$ is congruent to a set $T$. $T$ is called the prototile of $\mathcal{T}$. We say that $T$ admits the tiling $\mathcal{T} . T$ is also called a space filler.

Let $K$ be a topological space. An embedding of $K$ in $\mathbb{R}^{3}$ is a homeomorphism $h$ from $K$ into $\mathbb{R}^{3} . K_{1}, K_{2} \subset \mathbb{R}^{3}$ are of the same embedding type if there is a homeomorphism $f: \mathbb{R}^{3} \rightarrow \mathbb{R}^{3}$ such that $f\left(K_{1}\right)=K_{2}$. Two spaces $K_{1}, K_{2}$ are of the same topological type if they are homeomorphic. Let $S \subset \mathbb{R}^{3}$ be a 2-sphere. $S$ is tame or tamely embedded if $S$ and the standard 2-sphere are of the same embedding type. Otherwise, $S$ is wild or wildly embedded. For a compact polyhedron $K$, not necessarily Euclidean, an embedding $h$ of $K$ into $\mathbb{R}^{3}$ is tame if $h(K)$ is of the same embedding type as a polyhedron (see, e.g. $[\mathrm{BC}])$.

There has been interest in which topological shape in $\mathbb{R}^{3}$ admits a tiling of $\mathbb{R}^{3}$, or what can be a space filler. The following is a list of relevant work, including some on 
spaces other than $\mathbb{R}^{3}$ : [Ad1], [Ad2], [Ba], [CS], [De], [K1], [K2], [O], [Schm1], [Schm2] and $[\mathrm{Schu}$. The question can be broken into two. Different answers to any of them give space fillers of different shapes.

Question 1. Spaces of which topological types have an embedding into $\mathbb{R}^{3}$ that admits a tiling of $\mathbb{R}^{3}$ ?

Question 2. Given a space of a topological type, which embedding types have a representative that admits a tiling of $\mathbb{R}^{3}$ ?

The most general result on these questions was by Adams [Ad1], [Ad2]. Concurrently and independently, Kuperberg [K1] obtained a similar but less general result. Adams showed that for any compact polyhedral 3-submanifold of $\mathbb{R}^{3}$ with one boundary component (or with nonempty and connected boundary), there is a polyhedral submanifold of the same embedding type that admits a monohedral tiling of $\mathbb{R}^{3}$. In other words, the topological types realizable by compact 3-manifolds that can be embedded in $\mathbb{R}^{3}$, and with one boundary component, are valid answers to Question 1 . This is because such manifolds can be triangulated (see [Bi1] and [M1]), and the embeddings can be approximated by piecewise linear homeomorphisms [M2, p. 251]. Given such a topological type, the tame embedding types are valid answers to Question 2. As the tiles considered are tame, the attention has been focused on their knottedness. Adams' result implies that however a tamely embedded compact 3-manifold with one boundary component is knotted, there is still a polyhedral submanifold of $\mathbb{R}^{3}$ of the same embedding type that can serve as the prototile of a monohedral tiling. There are elaborate results on tilings with knotted tiles by Adams [Ad1], [Ad2], Kuperberg [K1], [K2], Oh [O] and Schmitt [Schm1], [Schm2].

In this article we use the ideas and techniques developed by Adams, Kuperberg and Schmitt to exhibit two new classes of space fillers of $\mathbb{R}^{3}$. They are wildly embedded. We introduce some more terminologies.

Let $S$ be a 2 -sphere embedded in $\mathbb{R}^{3}$. Let Int $S$ and Ext $S$ be respectively the bounded and unbounded complementary domain of $S$. A crumpled cube is a space $K$ homeomorphic with $S \cup \operatorname{Int} S$ (see, e.g. [Bu] and [BC]). By the crumpled cube $S \cup \operatorname{Ext} S$, we mean a space homeomorphic to the image of $S \cup \operatorname{Ext} S$ under an inversion with the image of the point at infinity added. Sometimes, it is convenient to regard a crumpled cube as a topological type, containing all such spaces homeomorphic to one another. In this article, a 3-cell is a topological 3-cell. If a nontrivial crumpled cube (one other than the 3-cell) is embedded in $\mathbb{R}^{3}$, its boundary sphere must be wildly embedded. Crumpled cubes are compact, and are the closure of their interiors, and hence can be tiles under our consideration.

A crumpled cube can be topologically complicated. Here are some examples. Let $S$ be an Alexander horned sphere [Al] with $S \cup \operatorname{Int} S$ a 3-cell, as is usually drawn in $\mathbb{R}^{3}$. The fundamental group of the crumpled cube $S \cup \operatorname{Ext} S$ (E.T.'s bowling ball) is not finitely generated (see $[\mathrm{BF}]$ and $[\mathrm{C}]$ ). The same statement is true for the Antoine horned sphere [R]. Let $S$ be the version of the Fox-Artin wild sphere with one wild point [FA], [BC], [Bu], with $S \cup \operatorname{Int} S$ a 3-cell. The crumpled cube $S \cup \operatorname{Ext} S$ is not locally simply connected at the wild point. 
There are many crumpled cubes of different topological types. For example, for each $n \in \mathbb{N}$, let $S_{n}$ be a 2-sphere in $\mathbb{R}^{3}$ with $n$ Fox-Artin type wild points, with $S_{n} \cup \operatorname{Int} S_{n}$ a 3 -cell . Then the crumpled cubes $S_{n} \cup$ Ext $S_{n}$ are of different topological types, as they have different number of points where the space is not locally simply connected.

We state our results now.

Theorem 1.1. Let $K$ be a crumpled cube. Then there is an embedding $h$ of $K$ into $\mathbb{R}^{3}$ such that $h(K)$ admits a monohedral tiling of $\mathbb{R}^{3}$.

Theorem 1.1 gives new answers to Question 1. If a crumpled cube is homeomorphic to a compact polyhedral 3-submanifold of $\mathbb{R}^{3}$ with one boundary component, the known answers to the question, then it can be tamely embedded. That is possible only for the trivial crumpled cube, a 3-cell. We use an idea in [Ad1], [Ad2] and [K1] to prove the theorem.

Theorem 1.2. There is an (a specific embedding of the) Alexander horned sphere $S$, together with the topologically trivial complementary domain as Int $S$, that admits a monohedral tiling of $\mathbb{R}^{3}$.

Theorem 1.2 is addressed to Question 2. It says that even for the 3-cell, which cannot be knotted, there are embeddings of different embedding types that admit tilings of $\mathbb{R}^{3}$. This gives an answer to Question 2 in a direction different from those in previous works on knotted tiles [Ad1], [Ad2], [K1], [K2], [O], [Schm1], [Schm2]. We use the technique developed in these works to prove the theorem.

By growing solid horned spheres on compact polyhedral 3-submanifolds of $\mathbb{R}^{3}$ with one boundary component, we produce space fillers homeomorphic to these submanifolds but of different embedding types, differing in their tameness, not knottedness. This is the content of Theorem 1.3, again addressed to the second question. Condition (c) in the theorem indicates that the knottedness of the original manifold is preserved. Adams' argument in $[\mathrm{Ad} 2]$ is used in its proof.

Theorem 1.3. Given a compact polyhedral 3-submanifold $M$ of $\mathbb{R}^{3}$ with one boundary component, there is a subspace $M^{\prime}$ of $\mathbb{R}^{3}$ with the following properties:

(a) $M$ and $M^{\prime}$ are homeomorphic;

(b) $M$ and $M^{\prime}$ are of different embedding types;

(c) there are 2-disks $D \subset \partial M$ and $D^{\prime} \subset \partial M^{\prime}$ such that $\partial M \backslash D$ and $\partial M^{\prime} \backslash D^{\prime}$ are of the same embedding type;

(d) $M^{\prime}$ admits a monohedral tiling of $\mathbb{R}^{3}$.

By growing suitably embedded crumpled cubes instead of solid horned spheres on polyhedral submanifolds, we get even more answers to Question 1. See the remark at the end of the paper.

We prove Theorem 1.1 in Section 2. The solid horned sphere tile is exhibited in Section 3. Theorem 1.3 is proved in Section 4. 


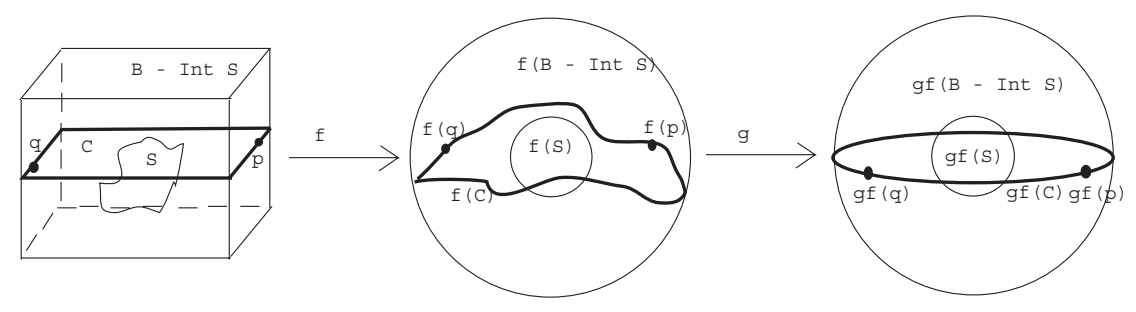

Fig. 1. Int $S$, Int $f(S)$ and $\operatorname{Int} g f(S)$ are not part of the diagram. $g f(B \backslash \operatorname{Int} S)$ is ready for further constructions.

\section{Tiling by Crumpled Cubes}

The proof of Theorem 1.1 uses an idea in [Ad1], [Ad2], and [K1] and the HosayLininger-Daverman theorem [Da], [H], [L]. The following is the form of the theorem to be used.

Hosay-Lininger-Daverman Theorem. Let $K$ be a crumpled cube. Then there is an embedding $h: K \rightarrow \mathbb{R}^{3}$ such that $\left[\mathbb{R}^{3} \backslash h(K)^{\circ}\right] \cup\{\infty\}$ is a 3 -cell.

Proof of Theorem 1.1. We present the proof in three steps.

Step 1 . Let $K$ be a crumpled cube. By the Hosay-Lininger-Daverman theorem, there is an embedding $h(K)$ such that $\left[\mathbb{R}^{3} \backslash h(K)^{\circ}\right] \cup\{\infty\}$ is a 3-cell. Let $S:=\partial h(K)$. Then $h(K)=S \cup \operatorname{Int} S$.

See Fig. 1. Let $B$ be a closed cube with $(S \cup \operatorname{Int} S) \subset B^{\circ}$. Let $C$ be the "equator" of $\partial B$, equally distanced from the top and the bottom of $B$ as drawn. Let $p, q \in C$ be chosen on opposite faces of $B$, so that when we glue together two copies of $B$, identifying the face containing $p$ of one copy and the face containing $q$ of the other copy, the points $p$ and $q$ of the two copies do not coincide.

Let $\Sigma$ be the shell $\left\{x \in \mathbb{R}^{3}, 1 \leq\|x\| \leq 2\right\}$. Let $\Sigma_{1}$ and $\Sigma_{2}$ be the inner and outer component of $\partial \Sigma$. As $\left(\mathbb{R}^{3} \backslash \operatorname{Int} S\right) \cup\{\infty\}$ is a 3-cell, $B \backslash$ Int $S$ is homeomorphic to $\Sigma$. Let $f: B \backslash$ Int $S \rightarrow \Sigma$ be a homeomorphism.

As $f(C)$ is a simple closed curve on $\Sigma_{2}$, it is tame [M2, p. 71]. Hence there is a homeomorphism $\varphi: \Sigma_{2} \rightarrow \Sigma_{2}$ such that $\varphi(f(C))$ is a great circle on $\Sigma_{2}$. Extend $\varphi$ to a homeomorphism $g: \Sigma \rightarrow \Sigma$, by defining $g(x)=(\|x\| / 2) \varphi(2 x /\|x\|)$ for $x \in \Sigma$.

Step 2. See Fig. 2. Let $A$ be an annulus with $g(f(C))$ as a component of its boundary, $A:=\left\{t x: x \in g(f(C)), t \in\left[\frac{1}{2}, 1\right]\right\}$. Let $D_{p}, D_{q} \subset \Sigma_{2}$ be disjoint closed disks centered at $g(f(p))$ and $g(f(q))$ such that $f^{-1} g^{-1}\left(D_{p}\right)$ and $f^{-1} g^{-1}\left(D_{q}\right)$ on adjacent copies of the cube are disjoint (as in Fig. 3). Construct two tubes $T_{p}, T_{q}$ joining $\Sigma_{1}$ and $\Sigma_{2}$ :

$$
\begin{aligned}
& T_{p}:=\left\{t x: x \in D_{p}, t \in\left[\frac{1}{2}, 1\right]\right\}, \\
& T_{q}:=\left\{t x: x \in D_{q}, t \in\left[\frac{1}{2}, 1\right]\right\} .
\end{aligned}
$$

The annulus $A$ divides $\Sigma \backslash\left(T_{p} \cup T_{q}\right)$ into two parts, with closures denoted $B_{1}$ and $B_{2}$. They are 3-cells. 

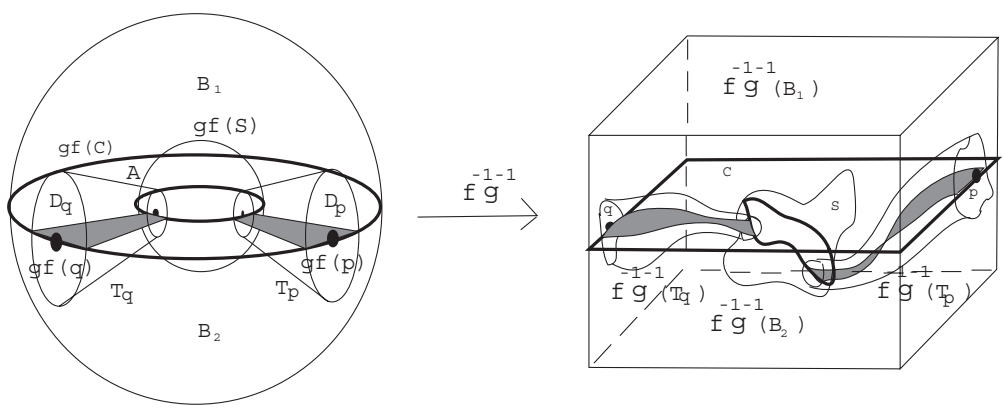

Fig. 2. Again Int $S$ is not part of the picture. The grey pieces are the intersections of the tubes with the annulus $A$, and their images under $f^{-1} g^{-1}$. $A$ and $f^{-1} g^{-1}(A)$ are not all shaded.

Step 3. Transplant the construction back to $B \backslash$ int (S) (Fig. 2). $B$ is decomposed into the crumpled cube $S \cup \operatorname{Int} S$, the tubes $f^{-1} g^{-1}\left(T_{p}\right)$ and $f^{-1} g^{-1}\left(T_{q}\right)$ and the 3-cells $f^{-1} g^{-1}\left(B_{i}\right), i=1,2$. Their interiors are disjoint.

Place copies of $B$ in a row (see Fig. 3). The prototile we are after consists of an embedded crumpled cube $(S \cup$ Int $S)$, the tubes $f^{-1} g^{-1}\left(T_{p}\right)$ and $f^{-1} g^{-1}\left(T_{q}\right)$ attached to it, the 3-cells $f^{-1} g^{-1}\left(B_{1}\right)$ on the right and $f^{-1} g^{-1}\left(B_{2}\right)$ on the left. Three copies of $f$ and $g$ are involved, but no confusion should be caused. The tile is homeomorphic to $S \cup \operatorname{Int} S$.

\section{A Solid Horned Sphere Tile}

Proof of Theorem 1.2. We present a solid horned sphere tiling of a cube, and hence $\mathbb{R}^{3}$, in four steps. The crucial point is that though the complement of a horned sphere in a quarter-cube is topologically complicated, it can be decomposed into three tame 3 -cells.

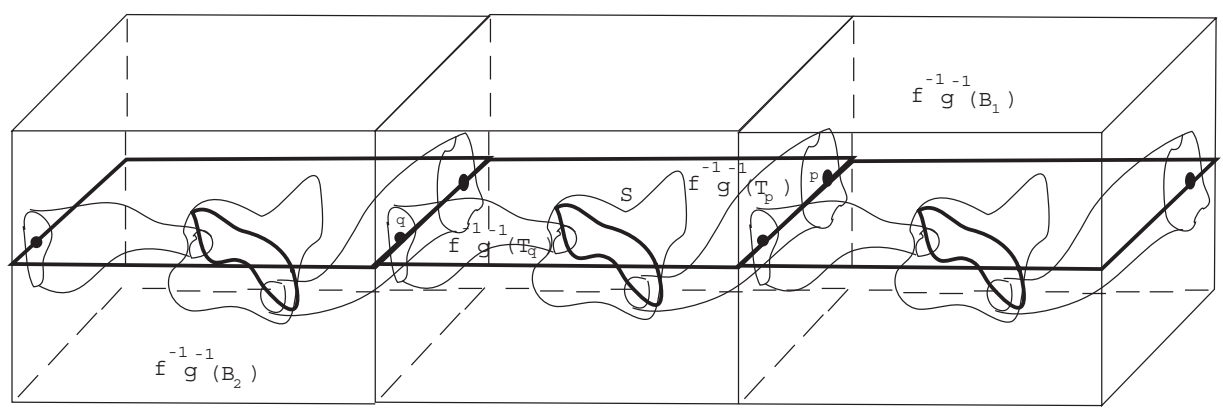

Fig. 3. Int $\mathrm{S}$ is part of the diagram. The prototile is the union of the crumpled cube $K=(S \cup$ Int $S), f^{-1} g^{-1}\left(T_{p}\right), f^{-1} g^{-1}\left(T_{q}\right)$ and $f^{-1} g^{-1}\left(B_{i}\right), i=1,2$, in three adjacent cubes as annotated. $f^{-1} g^{-1}(A)$ is not shaded. The three copies of $f$ and $g$ should cause no confusion. 


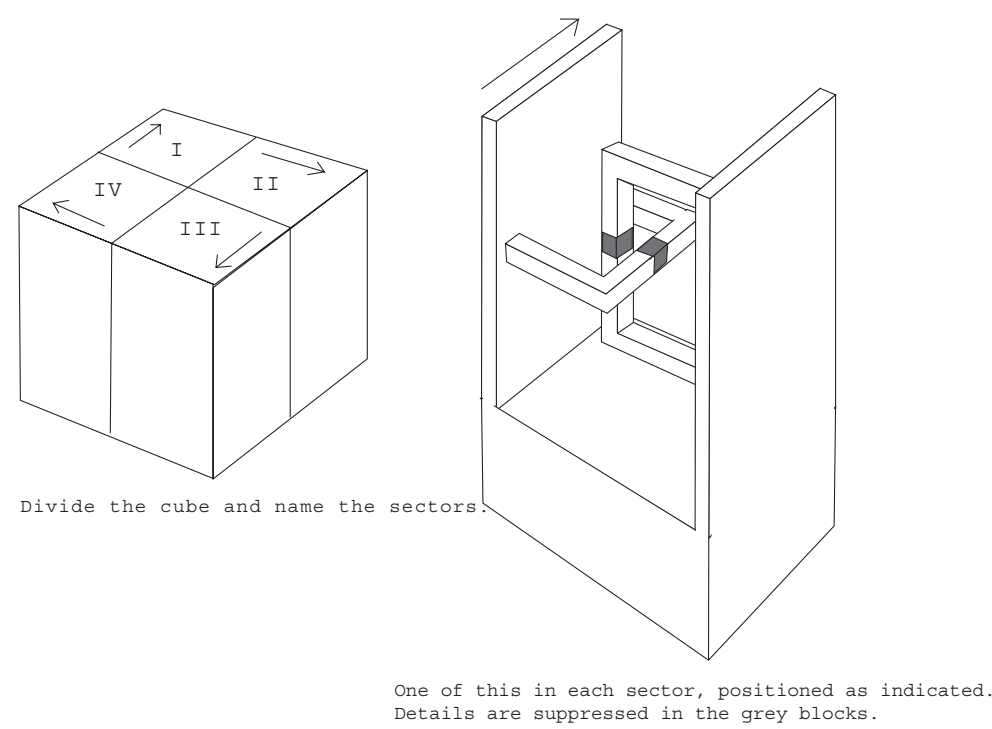

Fig. 4. The first step in constructing the solid horned sphere tile.

Step 1. Divide a cube into four sectors I, II, III, IV (see Fig. 4). In each sector we place a copy of a solid Alexander horned sphere with a topologically trivial interior, positioned as indicated. The details of the horned sphere are suppressed in the two grey small cubes. The complement of the horned sphere in a sector will be divided into three tame 3-cells. Steps 2 and 3 give the details.

Step 2. See Fig. 5. We divide a quarter-cube into nine layers. Layer i is ten times as thick as the others, and each of the other layers is divided into 81 small cubes as shown, viewed from above. They are of four colors: black (B), dark-grey (D), light-grey (L) and white $(\mathbf{W})$. The black small cubes form the bulk of the horned sphere. The small cubes and small half-cubes of the other colors form the bulk of the three 3-cells. The three special small cubes $A, B$ and $C$ are to be described later. There are small cubes which look redundant, but are needed later. This is explained below.

The small cube $B$ is divided into a thick $\mathbf{W}$ top part and a thin $\mathbf{L}$ bottom part (the $\mathbf{W}$ stuff will not form a cell later if the whole small cube $B$ is $\mathbf{W}$ ). Cubes $A$ and $C$ are constructed iteratively as follows.

Form a solid, called the iterating block, with small cubes labeled a-g, 2-8 and ii-viii but without the small cubes $A$ and $C$ (see Fig. 6(a)). It looks like a cube from the outside, but has two holes $(A$ and $C$ ) in it. (We now explain why some apparently redundant small cubes in Fig. 5 are needed. Layer ix, identical to layer viii, is needed for modification in step 4 . The half dark-grey block in layer iv is for the iterating block to look symmetric from the outside (Fig. 6). Small cubes with a coordinate equal to 2 or 3 are there to make the iterating block a cube. The rows $\mathrm{h}$ and $\mathrm{i}$ are there to make the sector look like a square when viewed from the above, so that the sector can be a quarter-cube. Layer i, ten times as thick as the other layers, is to make the height of the sector double its other dimensions so that it is a quarter-cube.) 


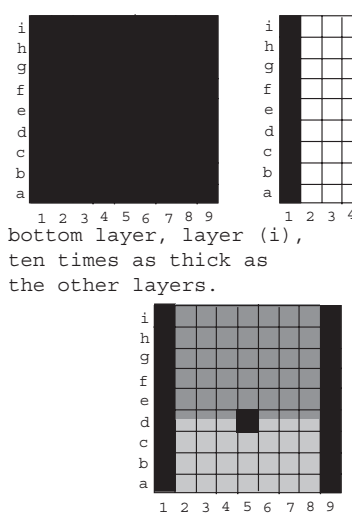

(vi)

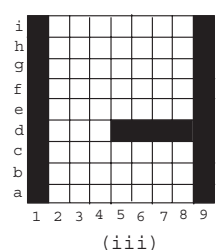

(iii)

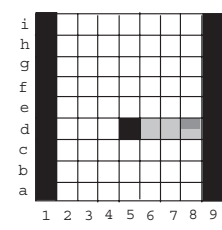

(iv)

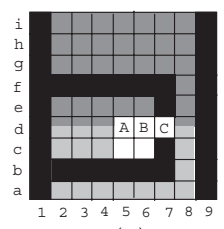

( $\mathrm{v})$ (ii)

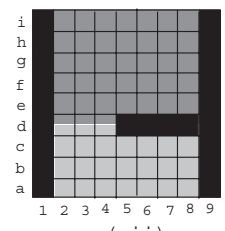

(vii)

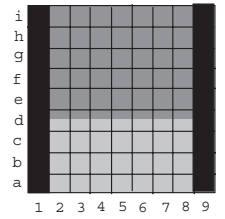

(viii)

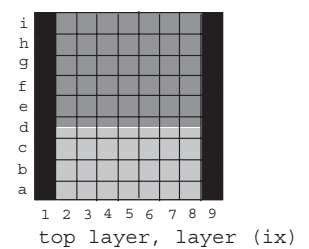

Fig. 5. The nine layers of a quarter-cube viewed from above. Each of them is subdivided into black (B), dark-grey (D), light-grey $(\mathbf{L})$ and white $(\mathbf{W})$ small cubes. Cubes $A, B, C$ are to be described. The $\mathbf{B}$ cubes formed the bulk of the solid horned sphere, while cubes of the other colors form the bulk of three 3-cells. Some apparently redundant small cubes are needed.

See Fig. 6(b). In cube $A$ place a shrunken and suitably rotated copy of the iterating block as indicated. A thin slice, of the same thickness of the light-grey slice in $B$, is cut away from the iterating block along the face facing down. The resulting gap is filled by expanding the black block iv-5-d below. Without doing this, the white material in $A$ will intersect the white material in the environment in two pieces: an edge of iv-5-e, and a 2-disk on the union of v-5-c, v-6-c, v-6-d (block $B$ ). Then the white material in the resulting solid will not form a 3-cell.

Do similar to cube $C$. Place a shrunken iterating block into $C$ as indicated (this is one of the two possible orientations). Cut away a thin slice of the iterating block along the face facing down. Expand the light-grey block iv-7-d below to fill the resulting space. Without doing this, the white material in the resulting solid will not be a 3-cell, as the white stuff in $C$ would intersect the original white stuff in three pieces: two line segments (on iv-7-c and iv-7-e) and one disk (along block $B$ ).

Iterate the process, filling the holes in the iterating blocks with even smaller iterating blocks with slices sliced away. As the iterating block is cubic, the horns and cubes added

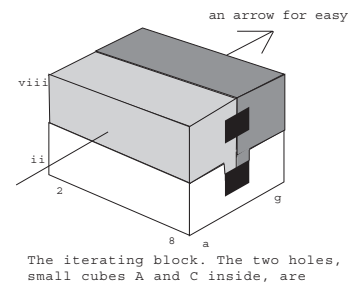

small cubes A and C Inside, are
hidden from view.

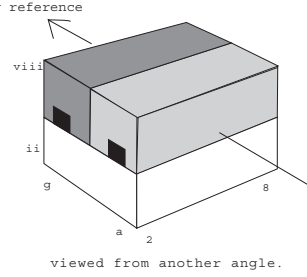

(a)

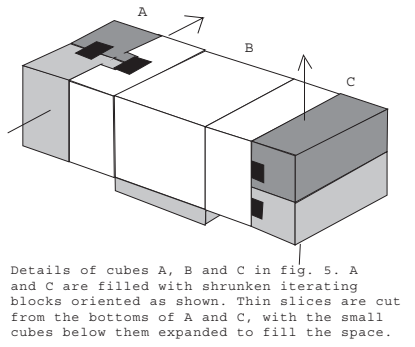

(b)

Fig. 6. The iterating block is used for constructing $A$ and $C$. (a) The iterating block. (b) Small cubes $A, B$ and $C$. 


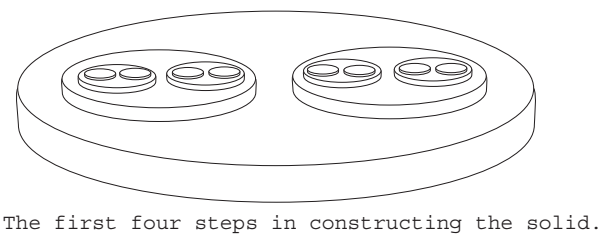

(a)

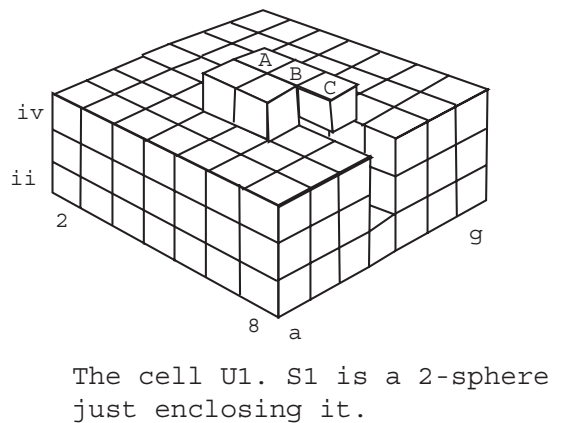

(b)

Fig. 7. (a) Each of the $\mathbf{D}, \mathbf{L}, \mathbf{W}$ materials in the finished product of the iteration form a figure homeomorphic to the solid indicated, a 3-cell. (b) The cell $U_{1}$ in the process of constructing $S_{1}$.

at different levels are similar, without flattening in any dimension. Look at the finished piece. It is clear that the black stuff form the solid horned sphere. The $\mathbf{W}, \mathbf{L}$ and $\mathbf{D}$ material form three 3-cells. Here is an explanation if it is needed. When we fill in blocks $A$ and $C$ iteratively, material of a color added in the $(n+2)$ th and later stages are at a distance from those already present in the $n$th stage. That is, the former stays away from the latter. Also the intersection of a component added in the $(n+1)$ th stage and the result of the $n$th stage is a 2 -disk. These guaranteed that the $\mathbf{W}, \mathbf{L}$ and $\mathbf{D}$ material in the finished piece are 3-cells. See Fig. 7(a).

Let $C_{\mathrm{D}}, C_{\mathrm{L}}$ and $C_{\mathrm{W}}$ be the dark-grey, light-grey and white 3-cell, respectively.

Step 3. We now show that the boundaries of the three 3-cells $C_{\mathrm{D}}, C_{\mathrm{L}}$ and $C_{\mathrm{W}}$ are tame. Without this step, we will still get a wildly embedded tile, only the tile may have more wild points than a solid horned sphere.

We say that a 2 -sphere $S \subset \mathbb{R}^{3}$ can be homeomorphically approximated from Int $S$ (Ext $S$ ) if for each $\varepsilon>0$, there are 2-spheres $S_{\varepsilon} \subset$ Int $S\left(S_{\varepsilon} \subset\right.$ Ext $S$ ) and homeomorphisms $h_{\varepsilon}: S_{\varepsilon} \rightarrow S$ that move no point more than $\varepsilon$. A 2-sphere $S$ in $\mathbb{R}^{3}$ is tame if and only if it can be homeomorphically approximated from Int $S$ and Ext $S$. See [Bi2] (or $[\mathrm{Bu}]$ or $[\mathrm{BC}])$.

We now show that $\partial C_{\mathrm{W}}$ is tame. As $C_{\mathrm{W}}$ is a cell, $\partial C_{\mathrm{W}}$ can be homeomorphically approximated from Int $S$. In Ext $S$ the sequence of approximations can be constructed as follows. The first approximating homeomorphism is constructed in two stages. We call the small cubes in Fig. 5 the first-level cubes. In the first stage take all the first-level cubes with interiors intersecting $C_{\mathrm{W}}$. They are the white cubes and cubes $A, B, C$. From the bottom of $A, B$ and $C$, discard a thin slice of uniform thickness containing no white stuff. The union of all these is called $U_{1}$ (Fig. 7(b)), which contains $C_{\mathrm{W}}$. It is a cell. Define a homeomorphism $f_{1}: \partial U_{1} \rightarrow \partial C_{\mathrm{W}}$ that moves points within the first-level cubes. Notice that this can be done, even in the trimmed cubes $A, B$ and $C$. Hence $f_{1}$ moves no point by more than $d_{1}$, the diameter of the first-level cubes. In the second stage let $S_{1}$ be a sphere with $U_{1} \subset$ Int $S_{1}$, and is very near to $\partial U_{1}$. Let $g_{1}: S_{1} \rightarrow \partial U_{1}$ be a homeomorphism moving points by less than $d_{1}$. Then the homeomorphism $h_{1}:=f_{1} \circ g_{1}: S_{1} \rightarrow \partial C_{\mathrm{W}}$ move points by less than $2 d_{1}$. 


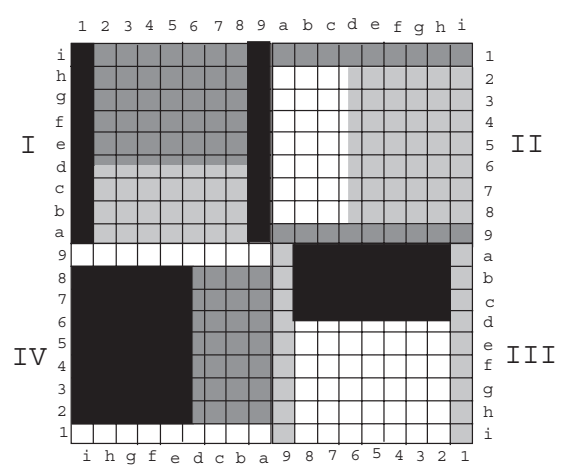

top layer, layer ix, before modification

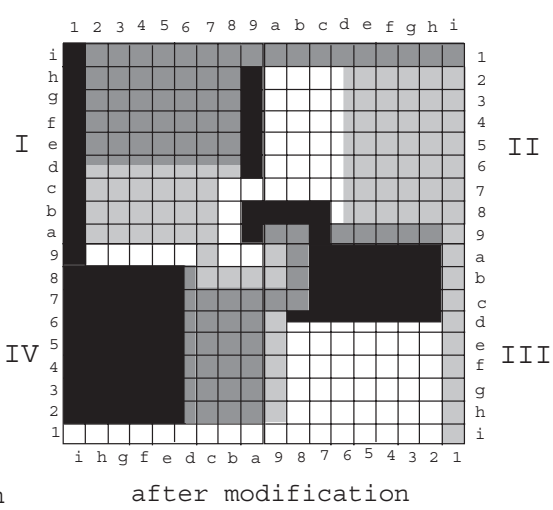

Fig. 8. Top layer of the four groups of horned sphere with three 3-cells.

Subdivide each first level cube into $7^{3}$ cubes, called the second-level cubes. They are of the same size as the small cubes in the iterating block shrunken once. Repeat the argument to get a homeomorphism $h_{2}$, which moves points by less than $2 d_{2}$, the diameter of the second-level cubes. Repeat the procedure to get the desired sequence of homeomorphisms. Hence $\partial C_{\mathrm{W}}$ can be homeomorphically approximated from the outside, and $C_{\mathrm{W}}$ is tame.

The same reasoning can be applied to cells $C_{\mathrm{D}}$ and $C_{\mathrm{L}}$. For $C_{\mathrm{L}}$, in constructing the corresponding $U_{1}$, one has to cut away a slice from the top of cubes $B$ and $C$, and a slice from cube $A$ adjacent to cube $B$. Then $\partial U_{1}$ can be mapped to $\partial C_{\mathrm{L}}$ by a homeomorphism moving points within first-level cubes, and hence by a distance $\leq d_{1}$. Then proceed as before.

Step 4. Four copies of the quarter-cube constructed in step 2 are packed into a cube, positioned as in Fig. 8, seen from above. Color the pieces so that as one goes clockwise through the sectors, the colors of the material are rotated as follows:

$$
\mathbf{B} \longrightarrow \mathbf{D} \longrightarrow \mathbf{L} \longrightarrow \mathbf{W} \longrightarrow \mathbf{B}
$$

Modify the top layer, layer ix, so that the 3-cells are attached to the horned spheres in different sectors as trivial topological extensions (see Fig. 8). Pieces of the same color are joined. Notice that another set of attachments occur below the surface, in layers ii, iii and iv. These new solid horned spheres are four congruent tiles tiling the cube.

Using a similar method, the Fox-Artin and other solid wild sphere space fillers can be constructed.

In the above proof, we have established a fact of independent interest.

Proposition 3.1. Let $S$ be an Alexander horned sphere in $S^{3}$. The closure of the topologically nontrivial complementary domain is the union of three tame 3-cells with mutually disjoint interiors. 
Proof. Using the construction in step 2 of the proof of Theorem 1.2, we have a rectangular block in $\mathbb{R}^{3}$ consisting of a solid horned sphere and three 3 -cells. Let the rest of $\mathbb{R}^{3}$, together with the point at infinity, be joined to the dark-grey cell.

The boundaries of any two of the three cells intersect in infinitely many pieces. Hence the combination of (refinements of) the triangulations of the cells will not be a triangulation of their union.

\section{Wild Tiles from Polyhedral Ones}

Using an argument similar to Adams' in [Ad2], we can produce many wildly embedded space-fillers by growing horned spheres on polyhedral submanifolds of $\mathbb{R}^{3}$ with one boundary component. Other wildly embedded cells can be used instead of the solid horned sphere. We first present Adams' theorem with an outline of the proof, slightly adapted for our purpose. We then use a similar argument to establish Theorem 1.3.

Adams' Theorem [Ad1], [Ad2]. Let M be a compact polyhedral 3-submanifold of $\mathbb{R}^{3}$ with one boundary component. Then a cube can be tiled with four polyhedral tiles, all of which are congruent and of the same embedding type as $M$.

Proof of Adams' Theorem Outlined. Place $M$ (shrunken if necessary) in the interior of a quarter-cube $Q$. Then $P:=\overline{Q \backslash M}$ is a polyhedral submanifold of $\mathbb{R}^{3}$. Decompose $P$ into three 3-cells as follows. Take a triangulation of $P$. Let $K$ be its 1 -skeleton, and let $G$ be the dual graph of $K$. Fatten them up to essentially disjoint closed sets $N_{K}$ and $N_{G}$ such that $N_{K} \cup N_{G}=P$. Two sets are said to be essentially disjoint if they have disjoint interiors. Let $G^{\prime}$ be a spanning tree of $G$. Let $N_{G^{\prime}}$ be a cell neighborhood of $G^{\prime}$ such that $N_{G}$ is the essentially disjoint union of $N_{G^{\prime}}$ and a number of other cells $C_{1}, \ldots, C_{\ell}$. Then $N_{K} \cup C_{1} \cup \cdots \cup C_{\ell}$ is a handlebody, which can be decomposed into two 3-cells. These cells together with $N_{G^{\prime}}$ give a decomposition of $P$ into three 3-cells.

By modifying these 3-cells if necessary, we can assume that the walls of $Q$ adjacent to the other quarter-cubes are within one of the cells. See Fig. 9(a). This is to ensure that the parts of the finished tile in different quarter-cubes will not be connected in a complicated way.

Place four identical groups of $M$ with three 3-cells into the quarter-cubes. Position the four groups so that rotations by integral multiples of $\pi / 2$ about the line of intersection of the four quarter-cubes are symmetries. From each of the four copies of $M$, run polyhedral tubes to connect it to three 3-cells, one in each of the other quarter-cubes, each one not the rotational images of the other two. The three-dimensional tubes are along the boundaries of the 3-cells and $M$, without piercing through them. That $M$ has only one boundary component guarantees that the cells are reachable from the copies of $M$. The result is four congruent figures, of the same embedding type as $M$. The figure is the polyhedral tile we want. 


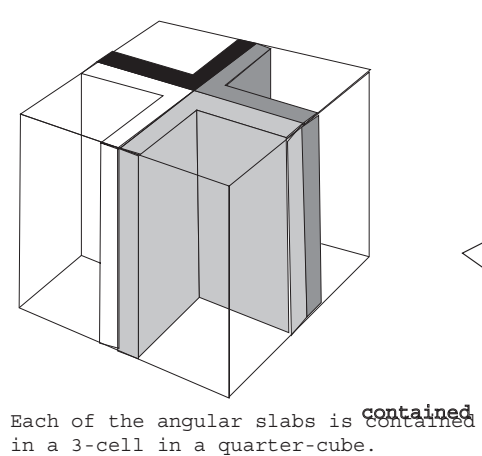

(a)

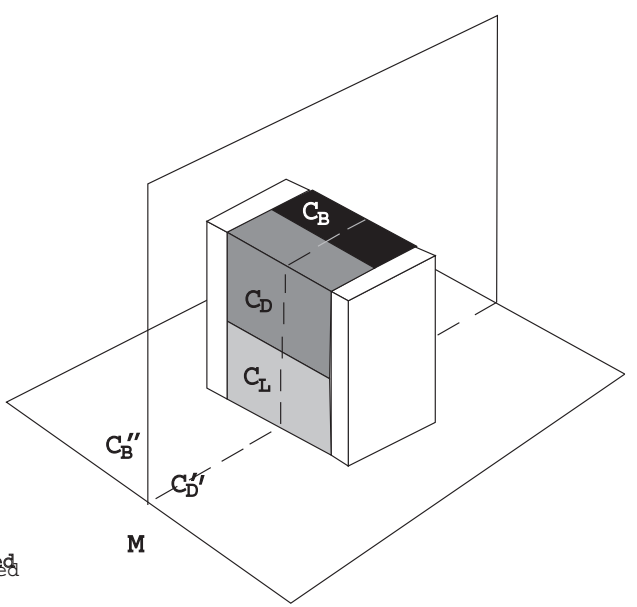

(b)

Fig. 9. (a) Four quarter-cubes. (b) A horned sphere block grown from a flat part of $M . C_{\mathrm{D}}^{\prime \prime}$ is $C_{\mathrm{D}}^{\prime}$ less the horned sphere block.

Proof of Theorem 1.3. Let $M$ be a compact polyhedral 3-submanifold of $\mathbb{R}^{3}$ with one boundary component. Let $Q$ be a quarter of the cube to be tiled. Place $M$ (shrunken if necessary) in $Q^{\circ}$. From the proof of Adams' theorem, $Q$ can be decomposed into $M$ and three polyhedral 3-cells. For convenience in drawing, $M$ is colored white, with the three cells named $C_{\mathrm{B}}^{\prime}, C_{\mathrm{D}}^{\prime}$ and $C_{\mathrm{L}}^{\prime}$ colored black, dark-grey and light-grey, respectively.

At least two of the cells $C_{\mathrm{B}}^{\prime}, C_{\mathrm{D}}^{\prime}$ and $C_{\mathrm{L}}^{\prime}$ intersect $\partial M$. Otherwise, the cell that intersect $\partial M$ encloses $M$ and hence fails to be a cell. Suppose that $C_{\mathrm{B}}^{\prime}$ and $C_{\mathrm{D}}^{\prime}$ intersect $\partial M$.

We can assume that there is a point $p \in \partial M \cap \partial C_{\mathrm{B}}^{\prime} \cap \partial C_{\mathrm{D}}^{\prime}$ but not in $C_{\mathrm{L}}^{\prime}$. Otherwise, we can modify the cells by transferring simplices of a (sufficiently fine) triangulation of $C_{\mathrm{L}}^{\prime}$ that form a polyhedral neighborhood of $p$ in $C_{\mathrm{L}}^{\prime}$, from $C_{\mathrm{L}}^{\prime}$ to $C_{\mathrm{B}}^{\prime}$ and $C_{\mathrm{D}}^{\prime}$ so that a neighborhood of $p$ does not intersect $C_{\mathrm{L}}^{\prime}$.

We can assume that $p$ is in the interior of a face of the polyhedral $M$. Otherwise, by modifying the cells through transferring simplices between $C_{\mathrm{B}}^{\prime}$ and $C_{\mathrm{D}}^{\prime}$, we can arrange that $M \cap C_{\mathrm{B}}^{\prime} \cap C_{\mathrm{D}}^{\prime}$ has some point in there.

See Fig. 9(b). On $\partial M$, in a neighborhood of $p$, we grow a white solid horned sphere with three tame 3 -cells $C_{\mathrm{B}}, C_{\mathrm{D}}, C_{\mathrm{L}}$, those we have constructed in step 2 of Section 3 except for color changes. The block does not intersect $C_{\mathrm{L}}^{\prime}$. The thick layer i is merged into $M$. (If the boundary between $C_{\mathrm{D}}^{\prime}$ and $C_{\mathrm{B}}^{\prime}$ is not vertical to $\partial M$ as drawn, slant the horned sphere block too.) Let $M^{\prime}$ be the union of $M$ and the solid horned sphere. Let $C_{\mathrm{B}}^{\prime \prime}$ and $C_{\mathrm{D}}^{\prime \prime}$ be respectively $C_{\mathrm{B}}^{\prime}$ and $C_{\mathrm{D}}^{\prime}$ less the horned sphere block (the horned sphere with $C_{\mathrm{B}}, C_{\mathrm{D}}$ and $C_{\mathrm{L}}$ ). They are 3-cells.

Decompose the complement of $M^{\prime}$ in the quarter-cube $Q$ into three tame 3-cells as follows. $C_{\mathrm{B}} \cup C_{\mathrm{B}}^{\prime \prime}$ and $C_{\mathrm{D}} \cup C_{\mathrm{D}}^{\prime \prime}$ give two tame 3-cells. Now $C_{\mathrm{L}}^{\prime}$ is disconnected from $C_{\mathrm{L}}$. Construct the third 3-cell $C_{\mathrm{L}}^{*}$ by joining $C_{\mathrm{L}}$ and $C_{\mathrm{L}}^{\prime}$ with a polyhedral tube along $\partial\left(C_{\mathrm{B}} \cup C_{\mathrm{B}}^{\prime \prime}\right), \partial\left(C_{\mathrm{D}} \cup C_{\mathrm{D}}^{\prime \prime}\right)$ or $\partial M^{\prime}$, modifying $C_{\mathrm{B}} \cup C_{\mathrm{B}}^{\prime \prime}, C_{\mathrm{D}} \cup C_{\mathrm{D}}^{\prime \prime}$ and/or $M^{\prime}$ along the way. This can be done, as $C_{\mathrm{L}}^{\prime}$ contacts either $M, C_{\mathrm{B}}^{\prime}$ or $C_{\mathrm{D}}^{\prime}$, and $\partial\left(C_{\mathrm{B}} \cup C_{\mathrm{B}}^{\prime \prime}\right), \partial\left(C_{\mathrm{D}} \cup C_{\mathrm{D}}^{\prime \prime}\right)$ and $\partial M^{\prime}$ are connected. 
Proceed as in the last part of the proof of Adams' theorem. Each quarter-cube is decomposed into a copy of $M^{\prime}$ and three tame 3-cells as in the last paragraph. They are positioned so that rotation by integral multiples of $\pi / 2$ about the line of intersection of the quarter-cubes are symmetries. Color the pieces so that as one rotates through $\pi / 2$, cells and $M^{\prime}$ are rotated into congruent copies, of different colors. 3-Cells of the same color are not connected, as a consequence of the construction shown in Fig. 9(a). From each of the four copies of $M^{\prime}$, run polyhedral tubes (also mapped into each other by rotations) to three cells of the same color, one in each of the other quarter-cubes, each not a rotational image of the others. This is the tile we are after, four of which tile a cube.

Remark. Instead of growing a solid horned sphere with three 3-cells on a compact 3 -submanifold with one boundary component $M$, we can grow the cube in Fig. 2: a suitably embedded crumpled cube (including the two tubes) and two 3-cells. If $M$ is not a cell, its union with a crumpled cube cannot be homeomorphic to the crumpled cube, as its boundary is not a sphere. Hence we get more space fillers of new topological types.

\section{References}

[Ad1] C. C. Adams, Tilings of space by knotted tiles, Math. Intelligencer, 17(2) (1995), 41-51.

[Ad2] C. C. Adams, Knotted tillings, in The Mathematics of Long-Range Aperiodic Order (R. V. Moody, ed.), pp. 1-8, Kluwer Academic, Dordrecht, 1997.

[Al] J. W. Alexander, An example of a simply connected surface bounding a region which is not simply connected, Proc. Nat. Acad. Sci. U.S.A., 10 (1924), 8-10.

[Ba] T. F. Banchoff, Torus decompositions of regular polytopes in 4-space, in Shaping Space-A Polyhedral Approach (M. Senechal and G. Fleck, eds.), pp. 221-230, Birkhäuser, Basel, 1988.

[Bi1] R. H. Bing, Locally tame sets are tame, Ann. of Math., 59 (1954), 145-158.

[Bi2] R. H. Bing, Conditions under which a surface in $\mathbb{E}^{3}$ is tame, Fund. Math., 47 (1959), 105-139.

$[\mathrm{BF}]$ W. A. Blankinship, R. H. Fox, Remarks on certain pathological open subsets of 3-space and their fundamental groups, Proc. Amer. Math. Soc., 1 (1950), 618-624.

[Bu] C. E. Burgess, Embedding of surfaces in Euclidean three-space, Bull. Amer. Math. Soc., 81 (1975), 795-818.

[BC] C. E. Burgess, J. W. Cannon, Embeddings of surfaces in $\mathbb{E}^{3}$, Rocky Mountain J. Math., 1 (1971), 259-344.

[C] R. P. Coelho, On the groups of certain linkages, Portugal. Math., 6 (1947), 57-65.

[CS] H. S. M. Coxeter, G. C. Shepard, Regular 3-complexes with toroidal cells, J. Combin. Theory Ser. $B, 22$ (1977), 131-138.

[Da] R. J. Daverman, A new proof of the Hosay-Lininger theorem about crumpled cubes, Proc. Amer. Math. Soc., 23 (1969), 52-54.

[De] H. E. Debrunner, Tiling three-space with handlebodies, Studia Sci. Math. Hungar, 21 (1986), 201202.

[FA] R. H. Fox, E. Artin, Some wild cells and spheres in three dimensional spaces, Ann. of Math., 49 (1948), 979-990.

[GS] B. Grunbaum, G. C. Shephard, Tilings and Patterns, Freeman, San Francisco, CA, 1987.

[H] N. Hosay, The sum of a real cube and a crumpled cube is $S^{3}$, Notices Amer. Math. Soc., 10 (1963), 666; errata 11 (1964), 152.

[K1] W. Kuperberg, Knotted lattice-like space fillers, Discrete Comput Geom., 13 (1995), 561-567.

[K2] W. Kuperberg, Tiling the solid torus, the 3-cube and the 3-sphere with congruent knotted tori, Intuitive Geometry (Budapest, 1995), pp. 399-406, Bolyai Society Mathematical Studies, Vol. 6, Janos Bolyai Mathematical Society, Budapest, 1997. 
[L] L. L. Lininger, Some results on crumpled cubes, Trans. Amer. Math. Soc., 118 (1965), 534-549.

[M1] E. Moise, Affine structures in 3-manifolds, VIII. Invariance of knot-type; local tame imbedding, Ann. of Math., 59 (1954), 159-170.

[M2] E. Moise, Geometric Topology in Dimensions 2 and 3, Springer-Verlag, New York, 1977.

[O] S. Oh, Knotted solid tori decomosition of $B^{3}$ and $S^{3}$, J. Knot Theory Ramifications, 5(3) (1996), 405-416.

[R] T. B. Rushing, Topological Embeddings, Academic Press, New York, 1973.

[Schm1] P. Schmitt, Another space-filling trefoil knot, Discrete Comput Geom., 13 (1995), 603-607.

[Schm2] P. Schmitt, Space filling knots, Beiträge Algebra Geom., 38(2) (1997), 307-313.

[Schu] E. Schulte, Space fillers of higher genus, J. Combin. Theory Ser. A, 68 (1994), 438-453.

Received October 22, 2002, and in revised form May 29, 2003. Online publication February 13, 2004. 